\title{
Characterization of PfTrxR inhibitors using antimalarial assays and in silico techniques
}

\author{
Ranjith Munigunti ${ }^{1 \dagger}$, Symon Gathiaka ${ }^{2 \dagger}$, Orlando Acevedo ${ }^{2}$, Rajnish Sahu ${ }^{3}$, Babu Tekwani $^{3}$ and Angela I Calderón ${ }^{1 *}$
}

\begin{abstract}
Background: The compounds 1,4-napthoquinone (1,4-NQ), bis-(2,4-dinitrophenyl)sulfide (2,4-DNPS), 4-nitrobenzothiadiazole (4-NBT), 3-dimethylaminopropiophenone (3-DAP) and menadione (MD) were tested for antimalarial activity against both chloroquine (CQ)-sensitive (D6) and chloroquine (CQ)-resistant (W2) strains of Plasmodium falciparum through an in vitro assay and also for analysis of non-covalent interactions with $P$. falciparum thioredoxin reductase (PfTrxR) through in silico docking studies.

Results: The inhibitors of PfTrxR namely, 1,4-NQ, 4-NBT and MD displayed significant antimalarial activity with $I C_{50}$ values of $<20 \mu \mathrm{M}$ and toxicity against 3 T3 cell line. 2,4-DNPS was only moderately active. In silico docking analysis of these compounds with PfTrxR revealed that 2,4-DNPS, 4-NBT and MD interact non-covalently with the intersubunit region of the enzyme.
\end{abstract}

Conclusions: In this study, tools for the identification of PfTrxR inhibitors using phenotyphic screening and docking studies have been validated for their potential use for antimalarial drug discovery project.

Keywords: Malaria, Plasmodium falciparum, Thioredoxin reductase, Molecular modeling

\section{Background}

Malaria, a tropical parasitic disease, continues to be the dominant cause of death in low-income countries especially in Africa and is considered to be one of the top three killers among communicable diseases [1]. Malaria caused by Plasmodium falciparum is considered to be the most deadly and also the one with highest rate of drug resistance [2]. Research investment in new and improved interventions will improve malaria cure, control, increase the cost-effectiveness of interventions and support efforts to eliminate malaria [3].

$P$. falciparum requires efficient antioxidant and redox systems to prevent damage caused by reactive oxygen species. In recent years, it has been shown that $P$. falciparum $(P f)$ possesses a functional low molecular weight thiol thioredoxin (Trx) system [4]. Thioredoxin reductase (TrxR) is an important enzyme of this redox system that helps the parasite to maintain an adequate intracellular redox environment during intraerythrocytic

\footnotetext{
* Correspondence: aic0001@auburn.edu

†Equal contributors

${ }^{1}$ Department of Pharmacal Sciences, 4306 Walker Building, Auburn University, Auburn, AL, USA

Full list of author information is available at the end of the article
}

development and proliferation. This antioxidant enzyme (PfTrxR) is essential for the survival of Plasmodium parasites for combating intraerythrocytic oxidative stress. Disruption of this enzyme is a feasible way to interfere with intraerythrocytic development and proliferation of the malaria parasites [5]. The current chemotherapy for malaria as recommended by WHO focuses on artemisinin-based combination therapies (ACTs) as the front line of treatment for malaria disease. The main drawbacks of combination therapies are high cost, adverse drug reactions and a high degree of pharmacokinetic mismatch between components leading to prolonged exposure of parasites to low doses of partner drug and its active metabolites which may facilitate development of resistant parasites [6].

Development of parasites' resistance to the known antimalarials remains a major challenge for the effective management of malaria. Intensive drug discovery programs have aimed at developing new antimalarials or modifying current antimalarials to improve their efficacy and reduce evidence of resistance.

In silico molecular modeling methods, such as docking can aid in the drug discovery process by ascertaining the binding affinities of existing and hypothetical compounds

\section{(Chemistry Central}


towards PfTrxR and the human isoform of this enzyme. Ideally, the simulations can also elucidate the origin behind the observed inhibition, as crystalline enzyme/ inhibitor complexes of the thioredoxin protein for $\mathrm{x}$ ray structure determination have not been reported. A comparison with other disulfide reductases including glutathione reductases reveals the most common inhibitor binding sites are at the active site and at the crystallographic 2-fold axis in the large cavity at the dimer interface. These sites can be exploited for structure-based inhibitor development. The dimer interface shows noncompetitive or uncompetitive behavior and their interaction with the protein is purely non-covalent [7-10]. Docking calculations are well suited for exploration of this interface; however, the simulations are unable to reproduce covalent inhibitors that bind irreversibly at the active site. A combined experimental and computational effort may counterbalance this deficiency and provide an enhanced avenue for inhibitor development.

A comparison between the $h \mathrm{TrxR}$ and $P f \operatorname{TrxR}$ structures shows that they have $46 \%$ sequence identity and overlay with an RMSD of $0.91 \AA$ between the 374 monomer atom pairs. The most important difference that can be exploited for selective inhibition between the two enzymes is at the dimer interface. The interface in PfTrxR is narrower than in $h$ TrxR due to the presence of Tyr101 and His104 and can therefore host smaller molecules. Their counterparts in the human isoform are Gln72 and Leu75 and this difference can determine the chemical nature of suitable inhibitors [11]. The molecular surfaces of the parasite and the human enzymes also indicate that the charges on the cavity walls are different, with the $h$ TrxR's being more negatively charged compared to the PfTrxR's [11].

The current study is aimed to employ the combined approach of in silico molecular docking for identification of key interactions of $P f \operatorname{TrxR}$ inhibitors to improve selectivity and phenotypic antimalarial assays for identification of activity against susceptible and drug-resistant $P$. falciparum blood stage cultures to assure the identification of specific Pf TrxR inhibitors as scaffolds for lead optimization.

\section{Results and discussion}

The in vitro antimalarial activity of the five known inhibitors of PfTrxR (1,4-NQ, 2,4-DNPS, 4-NBT, 3-DAP, MD) [12,13] (Figure 1; Table 1) was evaluated against both CQ-sensitive (D6 clone) and CQ resistant (W2 clone) strains of $P$. falciparum, while cell cytotoxicity was determined against 3T3 cells (Table 1) using the procedure described earlier. The compounds 1,4-NQ and 4-NBT were found to be the most active against the two strains of $P$. falciparum, MD and 2,4-DNPS were moderately active, and 3-DAP was inactive. In terms of antiplasmodial activity against the W2 strain, 1,4-NQ

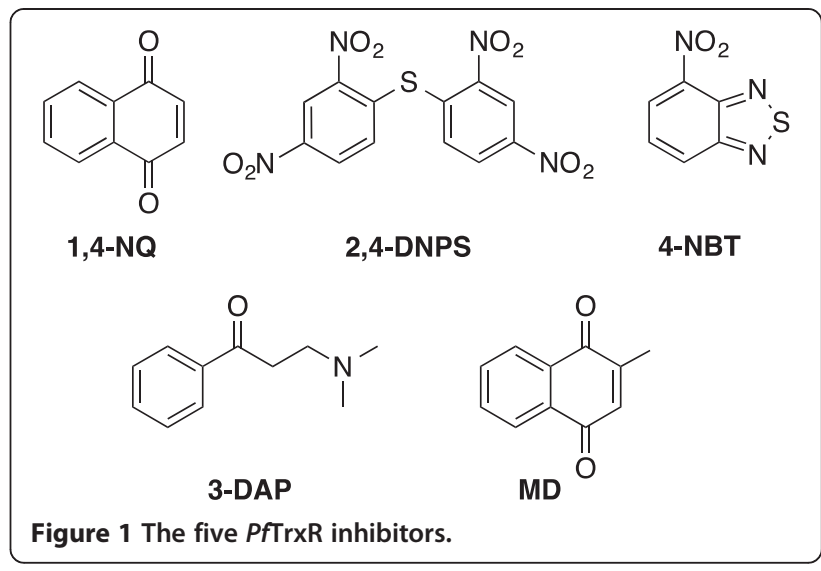

and $4-\mathrm{NBT}$ showed $\mathrm{IC}_{50}$ value of $<20 \mu \mathrm{M}$. The low correlation between the high PfTrxR inhibitory activity and moderate antiplasmodial activity of 2,4-DNPS could be explained by an inability to penetrate the cell membranes. Accordingly, 2,4-DNPS is predicted to have poor Caco-2 and MDCK cell line permeability. Table 2 gives the computed octanol/water partition coefficient $(\log \mathrm{P})$, solubility in water $(\log \mathrm{S})$, polar surface area, and apparent Caco-2 and MDCK permeability for all compounds given in Figure 1, the enol form of 3-DAP, and CQ.

The lack of antiplasmodial activity of 3-DAP, a Mannich base, may be due to (i) non-specific alkylation of cellular thiol groups, and also (ii) due to the absence of active transport to red blood cells and parasites. The correlation between inhibition of PfTrxR in the enzyme inhibition assays and antiplasmodial activity in cell culture allows for a better evaluation of biological activities of inhibitor compounds. The active compounds namely, 1,4-NQ, 2,4DNPS, 4-NBT and MD showed more toxicity than 3-DAP against the 3T3 cell line. The 3T3 cells are epithelial cells that reflect toxicity against proliferating mammalian cells.

In order to test the five PfTrxR inhibitors for their ability to induce signs of oxidative stress by accelerated generation and accumulation of reactive oxygen intermediates (superoxide radical, hydroxyl radical and hydrogen peroxide) [15] the intraerythrocytic formation of ROS was monitored in real-time for 120 min with 2' 7'-dichlorofluorescein diacetate (DCFDA), a fluorescent ROS probe [16]. Among the compounds tested 4-NBT, $\mathrm{MD}$ and 1,4-NQ caused a significant increase in oxidative stress (Figure 2). Whereas, 3-DAP and 2,4-DPNS did not cause the production of ROS. These results suggest that 4-NBT, MD and 1,4-NQ compromises the capability of erythrocytes to scavenge reactive oxygen intermediates. The accumulated intra-erythrocytic oxidative stress by these compounds may be responsible for the inhibition of $h$ TrxR enzyme. The erythrocytes, our target cells, have higher capacity to produce oxidative stress than 3T3 cell line used for cytotoxicity assessment. 
Table 1 PfTrxR inhibitory and antiplasmodial activities of tested compounds

\begin{tabular}{|c|c|c|c|c|c|c|}
\hline Test compounds & PfTrxR IC ${ }_{50}(\mu \mathrm{M})^{*}$ & Pf (D6) CQ sensitive $I C_{50}(\mu M)^{* *}$ & SI D6 & $P f(\mathrm{~W} 2) \mathrm{CQ}$ resistance $\mathrm{IC}_{50}(\mu \mathrm{M})^{* *}$ & SI W2 & $3 \mathrm{~T}_{3} \mathrm{IC}_{50}(\mu \mathrm{M})$ \\
\hline 1,4-NQ & 0.75 & $8.9 \pm 2.3$ & 4.6 & $16.7 \pm 3.7$ & 2.4 & $38.5 \pm 0.76$ \\
\hline 2,4-DNPS & 0.5 & $91.2 \pm 11.3$ & 0.8 & $72.3 \pm 11.3$ & 1.0 & $79 \pm 3.51$ \\
\hline $4-N B T$ & 2 & $8.3 \pm 2.1$ & 10 & $9.8 \pm 1.9$ & 8 & $80 \pm 1.15$ \\
\hline 3-DAP & 15.4 & $>100$ & $>1$ & $>100$ & $>1$ & $>100$ \\
\hline MD & 1.6 & $18.5 \pm 1.9$ & 3.8 & $28.3 \pm 5.6$ & 2.5 & $70.5 \pm 3.69$ \\
\hline CQ & & $0.055 \pm 0.006$ & & $0.440 \pm 0.045$ & & NC \\
\hline
\end{tabular}

* $\mathrm{C}_{50}$ values, preparation of PfTrxR and optimal experimental conditions for the PfTrxR functional assay were reported in Andricopulo et al., 2006 [12]; Charvet et al., 2003 [13]; Munigunti et al., 2012 [14].

**Values are mean \pm S.D. of triplicate observations; SI: Selectivity index. NC: no cytotoxicity up to concentration much higher than the concentration responsible for its antiplasmodial activity.

The 1,4-NQ chemical features and the ability to generate $\mathrm{OH}$ suggest the proficiency in altering intracellular redox status [17]. The antimalarial naphthoquinones (1,4-NQ and MD) are believed to perturb the major redox equilibria of the targeted $P$. falciparum infected red blood cells, which might be removed by macrophages. This perturbation results in development arrest and death of the malaria parasite at the trophozoite stage [18].

Since these compounds were active against $P f \operatorname{TrxR}$ as well, molecular docking was used to study their interactions with PfTrxR to gain further insight into the mode of interaction for these molecules. MD [19], DNPS and 4NBT [20] have been proposed to bind at the intersubunit region in PfTrxR's. However, 1,4-NQ and 3-DAP bind to the reductase covalently precluding the use of docking calculations. For example, 1,4-NQ is an inhibitor of TrxR that behaves as a subversive substrate [19]. The compound 3-DAP inactivates TrxR by alkylating the C-terminal redox active catalytic Cys-Cys pair. This is achieved by the formation of a reactive $\alpha, \beta$-unsaturated ketone intermediate after it undergoes deamination in solution [13]. Therefore 3-DAP acts an alkylator. The calculations predict the same activity trend observed in the experimental $\mathrm{IC}_{50}$ values for the non-covalent inhibitors, 2,4-DNPS, $\mathrm{MD}$, and 4-NBT in PfTrxR (Table 3).

For the $P f \operatorname{TrxR} / \mathrm{MD}$ complex, pi stacking interactions are predicted to form between the inhibitor's phenyl ring and Tyr101 side chain ring. The backbone nitrogen of Met105 is in close proximity to the carbonyl group of MD; however, the predicted angle between $\mathrm{N}-\mathrm{H}$ and $\mathrm{O}$ of $85^{\circ}$ impedes hydrogen bonding. The molecule further forms hydrophobic interactions with the phenyl ring of Tyr116' and the side chains of Ile108 from both subunits. Similar to MD, 4-NBT's phenyl ring also has pi-pi stacking with Tyr101's phenyl ring, but forms hydrophobic interactions only with Ile108 from subunit B in the large cavity. The nitro group causes the molecule to twist subtly compared to MD in order to better interact with the electrostatic surface created by the peptide bond between His104 and Met105's and sulfur (Figure 3). Compared to the size of the cavity, MD and 4-NBT are small molecules and do not fully interact with most of the residues lining the wall of the dimer interface.

2,4-DNPS forms the only electrostatic interaction at a distance of $3.9 \AA$ with Asn481'. Pi stacking interactions are formed between one of the inhibitor's phenyl rings and Tyr101 side chain ring with the other phenyl ring of the molecule forming a parallel displaced pi stacking interaction with Tyr101' (subunit B). As with MD and 4-NBT, the side chains of Ile108 from both subunits form hydrophobic interactions with 2,4-DNPS. Most of the interactions the three molecules are forming with the proteins are with the intersecting helices between the two subunits of the enzymes. The experimental activities

Table 2 Predicted physico-chemical properties of the compounds

\begin{tabular}{llllll}
\hline Molecule & $\begin{array}{l}\text { log P octanol/ } \\
\text { water (M) }\end{array}$ & $\begin{array}{l}\text { log S aqueous } \\
\text { solubility (M) }\end{array}$ & $\begin{array}{l}\text { Polar surface } \\
\text { area (PSA) }\end{array}$ & $\begin{array}{l}\text { Apparent caco-2 } \\
\text { permeability (nm/sec) }\end{array}$ & $\begin{array}{l}\text { Apparent MDCK } \\
\text { permeability (nm/sec) }\end{array}$ \\
\hline 1,4 NQ & 0.486 & -0.764 & 57.199 & 954 & 470 \\
2,4 DNPS & 1.406 & -3.444 & 177.135 & 5 & 2 \\
4-NBT & 0.746 & -1.414 & 75.626 & 382 & 385 \\
3-DAP & 1.249 & -0.313 & 32.353 & 819 & 441 \\
3-DAP-enol & 1.800 & -1.170 & 25.296 & 842 & 454 \\
MD & 0.880 & -1.312 & 55.456 & 1225 & 616 \\
CQ & 4.276 & -3.585 & 24 & 1364 & 1862 \\
Range 95\% of drugs & $(-2.0 / 6.5)$ & $(-6.5 / 0.5)$ & $(7.0 / 330.0)$ & $(<25$ poor, $>500$ great) & $(<25$ poor, $>500$ great) \\
\hline
\end{tabular}




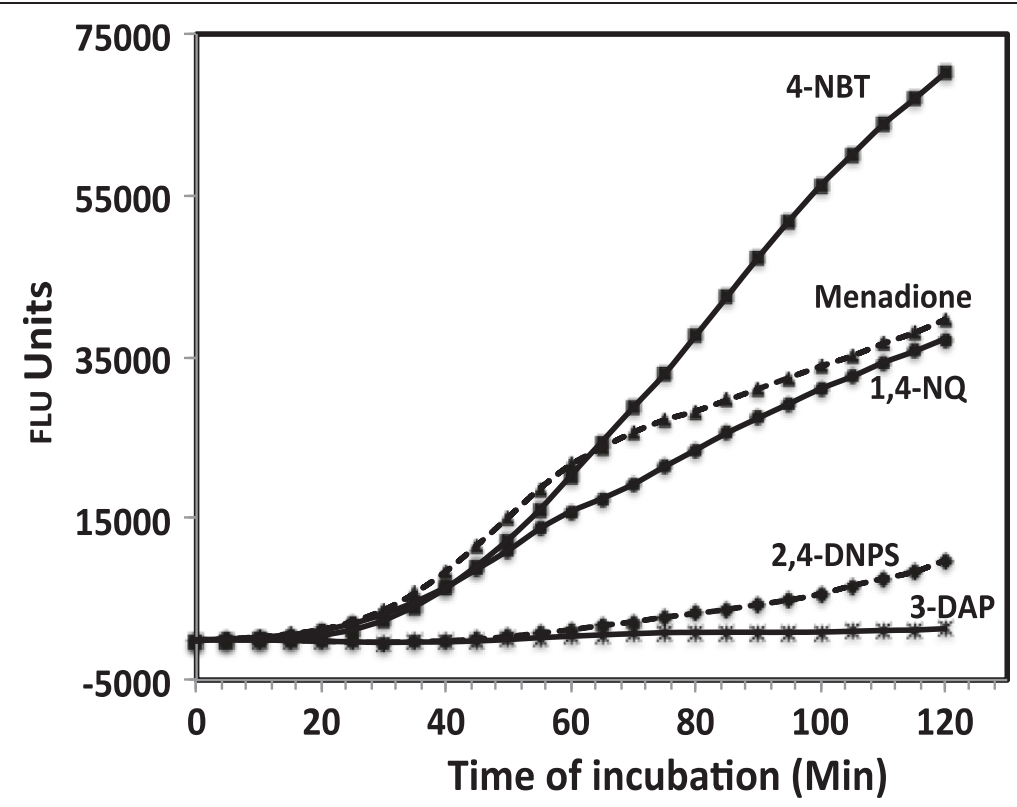

Figure 2 Formation of reactive oxygen species (ROS), as indicated by increase in fluorescence. DCFDA loaded human erythrocytes by five PfTrxR inhibitors.

for 2,4-DNPS and 4-NBT show selectivity between the parasite and human isoform of thioredoxin reductase (Table 4). The experimental values for 2,4-DNPS show an 8-fold selectivity for PfTrxR, whereas 4-NBT has a 25 -fold selectivity. While the docking simulations correctly predicted binding trends, limitations in the method, including potentially inaccurate scoring functions, the use of rigid proteins, and a lack of solvation could have contributed to its inability to reproduce the large differences observed in the $\mathrm{IC}_{50}$ values.

The docked poses, however, have considerable differences within the cavity, which could point to the observed selectivity (Figure 4). The presence of Tyr101 in PfTrxR enables 2,4-DNPS to form a favorable pi stacking interaction with the phenyl ring of the molecule, whereas its counterpart in $h \operatorname{TrxR}$ is a Gln72 that orients the molecule to avoid steric clashes. This results in the second ring of the molecule forming a parallel displaced pi stacking interaction with Tyr101', whereas a hydrogen bond between the nitro group and Gln72' in the $h$ TrxR is realized. The effect of this substitution on 4-NBT

Table 3 Comparison between computed binding affinities at the dimer interface in PfTrxR and experimental

$I_{50}$ values

\begin{tabular}{ccc}
\hline Molecule & $\begin{array}{c}\text { Computed binding } \\
\text { affinity }(\mathbf{k c a l} / \mathbf{m o l})\end{array}$ & Exptl. PfTrxR IC $\mathbf{~}_{\mathbf{5 0}}(\boldsymbol{\mu} \mathrm{M})$ \\
\hline 2,4-DNPS & -8.4 & 0.5 \\
MD & -7.9 & 1.6 \\
4-NBT & -6.0 & 2 \\
\hline
\end{tabular}

seems to be the fact that the presence of Gln72 pushes the molecule deep into the large cavity precluding the interaction with the residues of the intersecting helices between the subunits. 1,4-NQ and 4-NBT can be considered to be attractive leads for further optimization as these compounds display good Pf TrxR inhibitory and antiplasmodial activity.

A thorough examination of the residues making any form of interaction with the small molecules showed that no other, including His104 (Pf TrxR) and its counterpart in $h \operatorname{TrxR}$ (Leu75), influences the differences in binding between the parasite and human isoform. Figure 5 shows 2,4-DNPS docked in both proteins especially showing the positions of the His104 and Leu75 as an example.

\section{Conclusions}

In this study, tools for the identification of PfTrxR inhibitors using phenotypic screening and docking studies have been validated for their potential use for antimalarial drug discovery project.

\section{Experimental}

\section{Chemicals and enzymes}

Deionized water generated by a Milli-Q water system (Millipore, MA) was used in the experiments. All reagents were purchased from Sigma-Aldrich.

\section{Biological assays}

Antimalarial assay

Briefly, antimalarial activity of the compounds were determined in vitro on chloroquine sensitive (D6, Sierra 

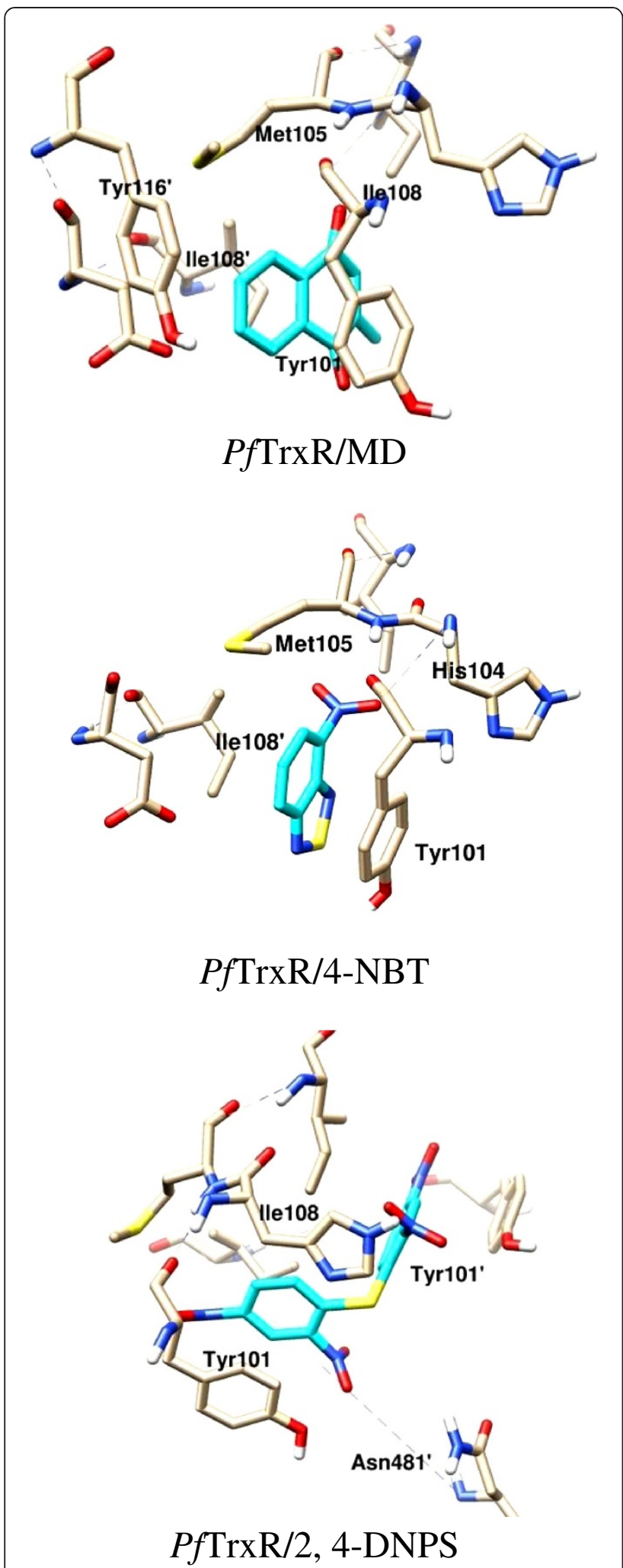

Figure 3 The predicted binding poses for the inhibitors showing the main interactions with the dimer interface residues. PfTrxR/MD, PfTrxR/4-NBT, and PfTrxR/2, 4-DNPS complexes.
Table 4 Comparison between computed binding affinities at the dimer interface and experimental $I C_{50}$ values in $P f$ TrxR and $h$ TrxR

\begin{tabular}{lccc}
\hline Molecule & & PfTrxR & hTrxR \\
\hline \multirow{2}{*}{ 2,4-DNPS } & Exptl. IC $50(\mu \mathrm{M})$ & 0.5 & 4 \\
& Calc. binding affinity $(\mathrm{kcal} / \mathrm{mol})$ & -8.4 & -8.1 \\
\multirow{2}{*}{ 4-NBT } & Exptl. IC & 2 & 50 \\
& Calc. binding affinity $(\mathrm{kcal} / \mathrm{mol})$ & -6.0 & -5.7 \\
\hline
\end{tabular}

Leone) and resistant (W2, IndoChina) strains of P. falcip arum. The 96-well microplate assay is based on the effect of the compounds on growth of asynchronous cultures of $P$. falciparum, as determined by the fluorometric SYBR green assay [21].

\section{Cytotoxicity assay}

Cytotoxicity in terms of cell viability was evaluated using 3 T3 cells by AlamarBlue assay [22]. This assay was conducted on compounds designated as active in the Pf TrxR functional assay and the antimalarial phenotypic screening.

\section{ROS assay}

Accelerated generation and accumulation of reactive oxygen intermediates (superoxide radical, hydroxyl radical

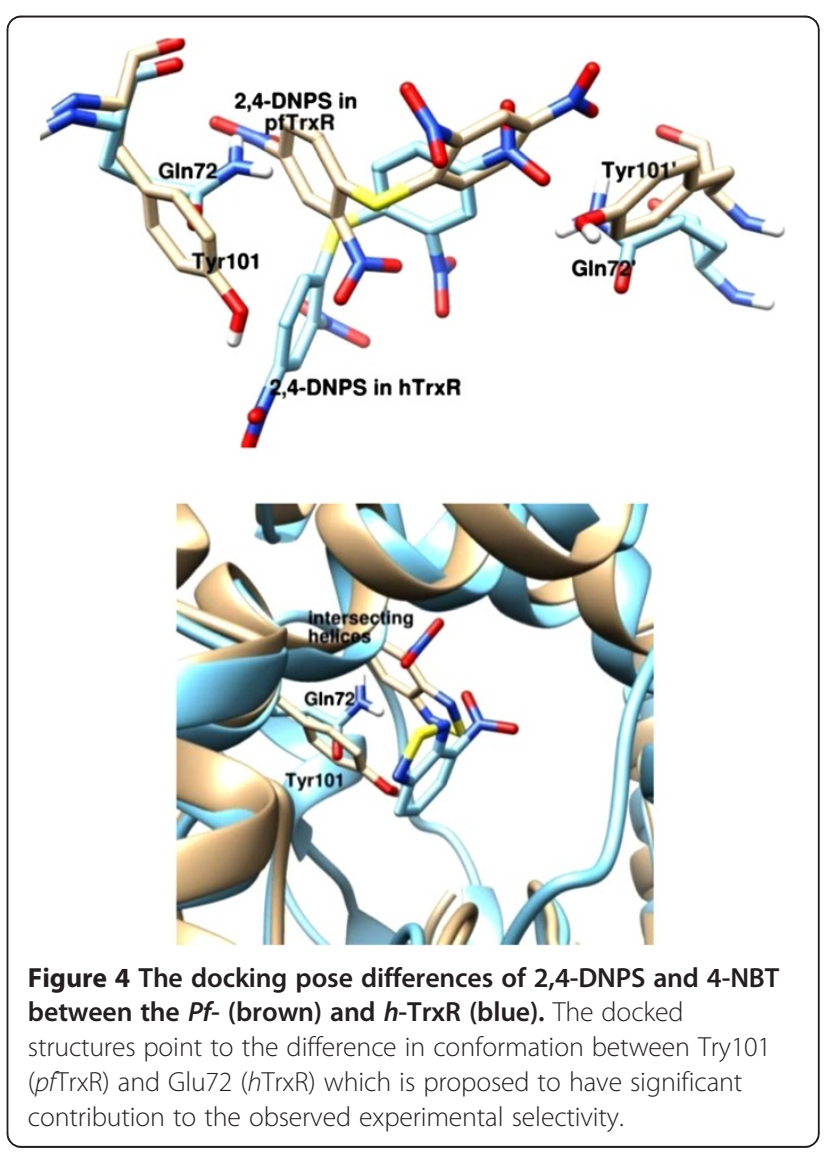




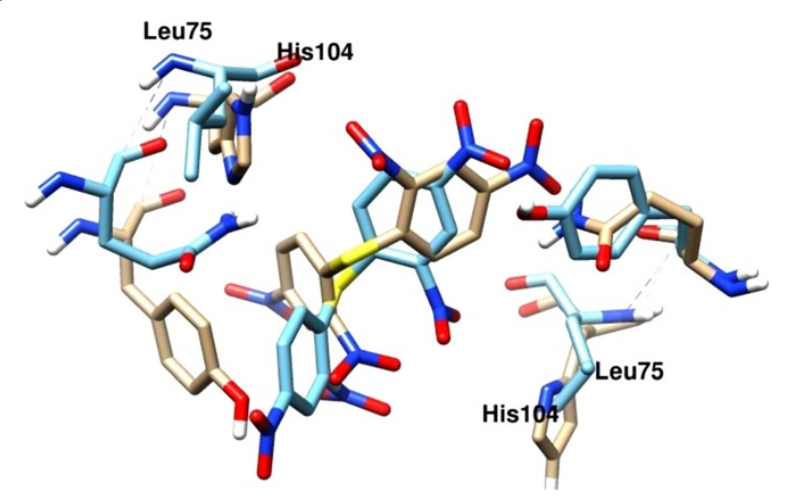

Figure 5 The predicted binding mode of 2,4-DNPS in the PfTrxR (beige) and $h$ TrxR (blue) showing the position of the residues Try101 (PfTrxR) and Glu72 ( $h$ TrxR).

and hydrogen peroxide) are mainly responsible for oxidative stress [15]. The intraerythrocytic formation of ROS was monitored in real-time with $2^{\prime} 7^{\prime}$-dichlorofluorescein diacetate (DCFDA), a fluorescent ROS probe [16]. Human erythrocytes collected in citrate phosphate anticoagulant were used. The erythrocytes were washed twice with $0.9 \%$ saline and suspended in PBSG at a hematocrit of $10 \%$. A $60 \mathrm{mM}$ stock of DCFDA was prepared in DMSO and added to the erythrocytes suspension in PBSG (10\% hematocrit) to obtain the final concentration of $600 \mu \mathrm{M}$. Erythrocytes suspension containing $600 \mu \mathrm{M}$ of DCFDA was incubated at $37^{\circ} \mathrm{C}$ for $20 \mathrm{~min}$ and centrifuged at $1000 \mathrm{~g}$ for $5 \mathrm{~min}$. The pellet of DCFDA loaded erythrocytes was suspended in PBSG to 50\% hematocrit and used for kinetic ROS formation assay. The assay was directly set up in a clear flat-bottom 96 well microplate. The reaction mixture contained $40 \mu \mathrm{l}$ of DCFDA loaded erythrocytes, the test compounds $(50 \mu \mathrm{M}))$ and potassium phosphate buffer (100 mM, pH 7.4), to make up the final volume to $200 \mu \mathrm{l}$. The controls without drug were also set up simultaneously. Each assay was set up at least in duplicate. The plate was immediately placed in a microplate reader programmed to kinetic measurement of fluorescence (excitation $488 \mathrm{~nm}$ and emission $535 \mathrm{~nm}$ ) for 2 hours with 5 min time intervals.

\section{Computational studies \\ Computational methods}

AutoDock Vina [22] was used to dock inhibitors to the respective targets. Initial Cartesian coordinates for the protein-ligand structures were derived from reported crystal structures of $h \operatorname{TrxR}$ (PDB ID: 3QFA) [23] and PfTrxR (PDB ID: 4B1B) [11]. The protein targets were prepared for molecular docking simulation by removing water molecules and bound ligands. AutoDockTools (ADT) [24] was used to prepare the docking simulations whereas Chimera was used to analyze the docking poses. All ligands were constructed using PyMol [25] with subsequent geometry optimizations carried out using the semi-empirical method PDDG/PM3 [20,26,27]. Polar hydrogens were added. ADME properties logP, logS, polar surface area, and apparent Caco-2 permeability for each ligand were computed using QikProp [28,29]. Conjugate gradient minimizations of the systems were performed using GROMACS [30]. A grid was centered on the catalytic active site region and included all amino acid residues within a box size set at $\mathrm{x}=\mathrm{y}=\mathrm{z}=20 \AA$.

\section{AutoDock Vina details}

Standard flexible protocols of AutoDock Vina using the Iterated Local Search global optimizer [31] algorithm were used to evaluate the binding affinities of the molecules and interactions with the receptors. All ligands and docking site residues, as defined by the box size used for the receptors, were set to be rotatable. Calculations were carried out with the exhaustiveness of the global search set to 100 , number of generated binding modes set to 20 and maximum energy difference between the best and the worst binding modes set to 5. Following completion of the docking search, the final compound pose was located by evaluation of AutoDock Vina's empirical scoring function where the conformation with the lowest docked energy value was chosen as the best.

\section{Competing interests}

All the authors declare that they have no competing interests.

\section{Authors' contributions}

AIC supervised all the research work. RM carried out the PfTrxR inhibitory assays and wrote the manuscript, which is part of his Ph.D. thesis. SG performed the docking experiments and helped writing the section. OA supervised the experiments and results of docking experiments. RS conducted the antimalarial and cytotoxicity assays. BT supervised the experiments and results of the antimalarial and cytotoxicity assays. All authors read and approved the manuscript.

\section{Acknowledgements}

We are deeply indebted to Dr. Katja Becker from the Justus-Liebig University, Germany for supplying the enzyme PfTrXR. NCNPR is partially supported by USDA-ARS cooperative scientific agreement.

\section{Author details}

'Department of Pharmacal Sciences, 4306 Walker Building, Auburn University, Auburn, AL, USA. ${ }^{2}$ Department of Chemistry and Biochemistry, Auburn University, Auburn, AL, USA. ${ }^{3}$ National Center for Natural Products Research \& Department of Pharmacology, Research Institute of Pharmaceutical Sciences, School of Pharmacy, University of Mississippi, University, MS, USA.

Received: 31 July 2013 Accepted: 5 November 2013 Published: 10 November 2013

\section{References}

1. Becker K, Hu Y, Biller-Andorno N: Infectious diseases - a global challenge. Int J Med Microbiol 2006, 296:179-185.

2. World Health Organization: World malaria report 2012; 2012. http://www.who. int/malaria/publications/world_malaria_report_2012/report/en/.

3. Roll Back Malaria: The global malaria action plan: for a malaria-free world. Geneva, Switzerland: Roll back Malaria Partnership; 2008. 
4. Müller S: Thioredoxin reductase and glutathione synthesis in Plasmodium falciparum. Redox Rep 2003, 8:251-255.

5. Müller S, Gilberger TW, Krnajski Z, Lüersen K, Meierjohann S, Walter RD: Thioredoxin and glutathione system of malaria parasite Plasmodium falciparum. Protoplasma 2001, 217:43-49.

6. Nosten F, White NJ: Artemisinin-based combination treatment of falciparum malaria. Am J Trop Med Hyg 2007, 77:181-192.

7. Savvides SN, Karplus PA: Kinetics and crystallographic analysis of human glutathione reductase in complex with a xanthene inhibitor. J Biol Chem 1996, 271:8101-8107.

8. Karplus PA, Pai EF, Schulz GE: Crystallographic study of the glutathione binding site of glutathione reductase at $0.3-\mathrm{nm}$ resolution. Eur J Biochem 1989, 178:693-703.

9. Schönleben-Janas A, Kirsch P, Mittl PR, Schirmer RH, Krauth-Siegel RL: Inhibition of human glutathione reductase by 10-arylisoalloxazines: crystalline, kinetic, and electrochemical studies. J Med Chem 1996, 39:1549-1554.

10. Becker K, Christopherson Rl, Cowden WB, Hunt NH, Schirmer RH: Flavin analogs with antimalarial activity as glutathione reductase inhibitors. Biochem Pharmacol 1990, 39:59-65.

11. Boumis G, Giardina G, Angelucci F, Bellelli A, Brunori M, Dimastrogiovanni D, Saccoccia F, Miele AE: Crystal structure of Plasmodium falciparum thioredoxin reductase, a validated drug target. Biochem Biophys Res Commun 2012, 425:806-811.

12. Andricopulo D, Akoachere MB, Krogh R, Nickel C, McLeish MJ, Kenyon GL, Arscott LD, Williams CH, Davioud-Charvet E, Becker K: Specific inhibitors of Plasmodium falciparum thioredoxin reductase as potential antimalarial agents. Bioorg Medicinal Chem Lett 2006, 16:2283-2292.

13. Charvet ED, McLeish MJ, Veine DM, Giegel D, Arscott LD, Andricopulo AD, Becker K, Muller S, Schirmer RH, Williams CH, Kenyon GL: Mechanism-based inactivation of thioredoxin reductase from Plasmodium falciparum by Mannich bases. implication for cytotoxicity. Biochemistry 2003, 42:13319-1333.

14. Munigunti R, Calderón Al: Development of liquid chromatography/mass spectrometry based screening assay for PfTrxR inhibitors using relative quantitation of intact thioredoxin. Rapid Commun Mass Spectrom 2012, 26:1-6.

15. Sivilotti ML: Oxidant stress and haemolysis of the human erythrocyte. Toxicol Rev 2004, 23:169-188.

16. Ganesan S, Chaurasiya ND, Sahu R, Walker LA, Tekwani BL: Understanding the mechanisms for metabolism-linked hemolytic toxicity of primaquine against glucose 6-phosphate dehydrogenase deficient human erythrocytes: Evaluation of eryptotic pathway. Toxicology 2012, 294:54-60.

17. Shang Y, Chen C, Li Y, Zhao J, Zhu T: Hydroxyl radical generation mechanism during the redox cycling process of 1,4-naphthoquinone. Environ Sci Technol 2012, 46:2935-2942.

18. Müller T, Johann L, Jannack B, Brückner M, Lanfranchi DA, Bauer H, Sanchez C, Yardley V, Deregnaucourt C, Schrével J, Lanzer M, Schirmer RH, DavioudCharvet E: Glutathione reductase-catalyzed cascade of redox reactions to bioactivate potent antimalarial 1,4-naphthoquinones - a new strategy to combat malarial parasites. J Am Chem Soc 2011, 133:11557-11571.

19. Morin T, Besset JC, Moutet M, Fayolle M, Bruckner M, Limosin D, Becker K, Davioud-Charvet E: The aza-analogues of 1, 4-naphthoquinones are potent substrates and inhibitors of plasmodial thioredoxin and glutathione reductases and of human erythrocyte glutathione reductase. Org Biomol Chem 2008, 6:2731-2742

20. Tubert-Brohman I, Guimarães CRW, Jorgensen WL: Extension of the PDDG/ PM3 semiempirical molecular orbital method to sulfur, silicon, and phosphorus. J Chem Theory Comput 2005, 1:817-823.

21. Co E-M, Dennull RA, Reinbold DD, Waters NC, Johnson JD: Assessment of malaria in vitro drug combination screening and mixed-strain infections using the malaria sybr green I-based fluorescence assay. Antimicrob Agents Chemother 2009, 53:2557-2563.

22. Hamalainen-Laanaya HK, Orloff MS: Analysis of cell viability using timedependent increase in fluorescence intensity. Anal Biochem 2012, 429:32-38.

23. Fritz-Wolf K, Kehr S, Stumpf M, Rahlfs S, Becker K: Crystal structure of the human thioredoxin reductase-thioredoxin complex. Nat Commun 2011, 2:383.

24. Morris GM, Huey R, Lindstrom W, Sanner MF, Belew RK, Goodsell DS, Olson AJ: AutoDock4 and AutoDockTools4: automated docking with selective receptor flexibility. J Comput Chem 2009, 30:2785-2791.

25. DeLano WL: The PyMOL molecular graphics system, Version 1.5.0.4: Schrödinger, LLC. San Carlos, CA, USA: DeLano Scientific; 2002.
26. Repasky MP, Chandrasekhar J, Jorgensen WL: PDDG/PM3 and PDDG/MNDO: improved semiempirical methods. J Comput Chem 2002, 23:1601-1622

27. Tubert-Brohman I, Guimarães CRW, Repasky MP, Jorgensen WL: Extensition of the PDDG/PM3 and PDDG/MNDO semiempirical molecular orbitial methods to the halogens. J Comput Chem 2003, 25:138-150.

28. QikProp: version 3.0, Schrödinger. New York, NY: LLC; 2006.

29. Duffy EM, Jorgensen WL: Prediction of properties from simulations: free energies of solvation in hexadecane, octanol, and water. J Am Chem Soc 2000, 122:2878-2888.

30. Hess B, Kutzner C, Spoel D, Lindahl E: GROMACS 4: algorithms for highly efficient, load-balanced, and scalable molecular simulation. J Chem Theory Comput 2008, 4:435-447.

31. Trott O, Olson AJ: AutoDock Vina: improving the speed and accuracy of docking with a new scoring function, efficient optimization and multithreading. J Comput Chem 2010, 31:455-461.

doi:10.1186/1752-153X-7-175

Cite this article as: Munigunti et al:: Characterization of PfTrxR inhibitors using antimalarial assays and in silico techniques. Chemistry Central Journal 2013 7:175

Publish with ChemistryCentral and every
scientist can read your work free of charge
"Open access provides opportunities to our
colleagues in other parts of the globe, by allowing
anyone to view the content free of charge."
W. Jeffery Hurst, The Hershey Company.
- available free of charge to the entire scientific community
- peer reviewed and published immediately upon acceptance
- cited in PubMed and archived on PubMed Central
- yours - you keep the copyright
submit your manuscript here:
http://www.chemistrycentral.com/manuscript/

